\title{
Enabling Semantic-Based Ubiquitous Learning in UPnP Network Environment
}

\author{
Atchara Rueangprathum, Somchai Limsiroratana, and Suntorn Witosurapot
}

\begin{abstract}
Although Universal Plug and Play (UPnP) network is aimed basically at home/ office service automation for general purposes, it can turn out to support for ubiquitous learning objective as well, if somewhat level of semantic capability will be involved. In this paper, we discuss how the UPnP network infrastructure should be polished with the Linked Data principle for enabling semantic-based ubiquitous learning services so that sharing digital contents can be done between heterogeneous devices in an effective manner, without the need of many distinct learning standards. In addition, we describe a case of simple quiz to illustrate how the Linked Data publishing of distributed multimedia resources can be utilized in our extended UPnP architecture.
\end{abstract}

Index Terms - Linked data, semantic web, ubiquitous mobile learning, UPnP.

\section{INTRODUCTION}

Smart home network technology has provided excellent opportunities for people to experience both local services in home/office automation and public services from the Internet accesses to premises. However, for the educational purposes, smart home network is often used to facilitate for the remote access of e-learning facility, but it is rarely utilized to provide for group-based learning among computers or mobile devices at homes or enterprises. This is crucially due to the lack of adequate systems and tools, which are indeed necessary for the arrangement of ubiquitous and mobile learning inside or outside premises.

Although a number of mobile learning systems have been researched to date (see [1] and Refs therein), they do not take advantages of existing ubiquitous network infrastructures designed for smart home into consideration. Therefore, their architectures are complex inevitably, due to the necessity for handling many basic (but crucial) issues, such as associating heterogeneous devices, discovering devices nearby, or enabling collaborative learning operations on a peer-to-peer (P2P) basis. We argue that the complexity of these architectures can be lessened by involving the support of pervasive network infrastructure, such as Universal Plug and Play (UPnP) [2], which is targeted for smart home/office service automation.

In addition, we encourage the exploitation of web-related technologies for many reasons. For instance, they are the technologies that are founded in the UPnP infrastructure for handling the problem of platform heterogeneity. Developers

Manuscript received May 5, 2014; revised July 25, 2014.

Atchara Rueangprathum, Somchai Limsiroratana, and Suntorn Witosurapot are with the Department of Computer Engineering, Prince of Songkla University, Thailand (e-mail: rueangprathum@gmail.com, somchai@coe.psu.ac.th,wsuntorn@coe.psu.ac.th). can then take advantages of deploying their applications via Web browsers, regardless of platform technology. More importantly, it is possible that the Semantic Web technology, such as that of Linked Data approach [3], can be involved for resource integration and generation in the Web-enabled UPnP environment. Then, learning resources and associated metadata can be reused, discovered, and interoperated among local UPnP devices via the Web infrastructure and protocols.

The contribution of this paper is a design of P2P-based mobile learning service that adopts existing UPnP network infrastructure for architectural simplification, and exploits Linked Data for semantic integration of learning resources. The remaining part of this paper is organized as follows. Section II gives a background of UPnP network environment and the Linked Data approach for lightweight semantics. Section III describes the proposed system architecture and functional component designs. Section IV provides the detail of our prototype and a simple use case. Finally, Section V concludes the paper.

\section{BACKGROUND}

\section{A. UPnP Network Environment}

Universal Plug and Play [2] is a well-recognized ubiquitous network infrastructure containing with features, such as zero-configuration for network startup, automatic detection of devices and services in the same local network, and seamless network connection on P2P basis. Dominant features are on the adoption of Service-oriented Architecture (SOA), Web technologies and Internet standards, i.e. TCP/IP and HTTP, to serve at the lower-layer of UPnP protocol stack (as illustrated in Fig. 1). Hence, web-based applications can be readily implemented at any UPnP devices for performing various tasks, such as interacting with the other networked UPnP devices, consuming UPnP services, or extending them for improved functionality.

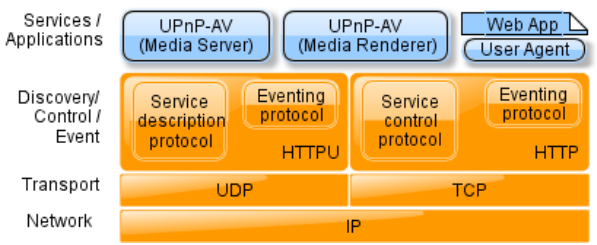

Fig. 1. Simplified UPnP protocol stack.

A notable UPnP extension is UPnP-AV [4], which enables ubiquitous multimedia services for home entertainments. Two extra-devices called Media Server and Media Renderer are introduced in UPnP-AV system architecture for specially managing Audio-Video (AV) content distribution on top of UPnP networks. The guideline in [5] suggests us how these 
special servers can be handled by User agent of UPnP device via HTML5 scripts in Web Applications for browsing and viewing the AV contents, or even managing to display them at the other device serving as Media Renderer. By working in this manner, it looks promising that UPnP-AV system can be augmented to share or distribute user-generated contents or resources among UPnP devices for learning purposes as well.

\section{B. Linked Data for Semantic-Based Learning Resources}

In order to provide adequate service for ubiquitous mobile learning, it is required that the all of learning contents must be involved not only with metadata for easing interoperability at the syntax level between devices, but also semantic-based operations for serving human-centric usability. In this regard, sharing and distributing multimedia contents in UPnP-AV system with MPEG-7 and MPEG-21 metadata standards are not adequate [5], especially in the aspects of learning resource with technologies of Learning Object. This is also applicable to the interoperability using popular educational metadata standards [6], e.g. IEEE-LOM and SCORM.

To accomplish the above requirement, traditional web pages crucially serving for human-centric operations will need to extend with semantic technology so that they can allow smart agent in a device to carry out tedious works, such as finding and combining learning resources on the Web, without human interventions. This requirement is indeed the vision of Semantic Web, but will be implemented by means of Linked Data approach [3], which is widely used to realize the Web of Data. It provides a smart way to expose, share, and reuse learning contents over the Web, thus giving benefits to lecturers or developers to create learning materials or quizzes in shorter period of time by combining contents from the other local devices or several public web sites.

From the technical aspect, the concept of Linked Data can be summarized by a) utilizing the Web as a single global database, b) describing the resource with the Resource Description Framework (RDF) annotation, and c) interlinking the data from different sources via Web addresses (URI). Once these resources are in RDF annotation, they become machine-readable, and hence give benefits to web developers in a number of ways. For instance, they can be viewed using generic data browsers, accessed via the links from the other resources, or even composed on-the-fly by smart agents residing at user-devices. It was noticed that the RDF data may be exposed as a standalone RDF or embedded into HTML documents via special attributes known as RDFa [7] for serving both machines and humans.

\section{EXTENDING UPNP-AV FOR UBIQUITOUS LEARNING}

This section illustrates the proposed architecture design that minimally extends the UPnP-AV architecture in such a way that ubiquitous mobile learning activities, either within a peer group or between a pair of devices, can be enabled.

\section{A. Design Abstraction}

In the provision of ubiquitous mobile learning services in the UPnP environments, the basic of UPnP-AV architecture will be taken into account (see Fig. 2) so that existing service entities can still be utilized, but realized in the new context.
For instance, the Media Server and Media Renderer remain dealing with MPEG-21 multimedia resources, which may be now alternately used as learning contents in some scenarios. Two exclusive services for handling educational resources are included into the system: a) Semantic data repository service working at a special server for storing and retrieving static learning resources, and b) Linked data service residing at the user-devices for manipulating and rendering linked data. These services can ensure that semantic interoperability of educational resources will be occurred properly in the system as explained in the previous section.

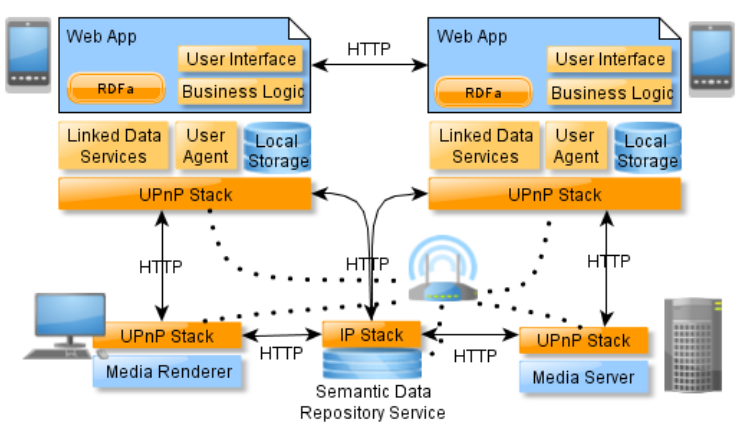

Fig. 2. Architecture overview.

\section{B. Semantic Data Repository Service}

Since some learning materials, e.g. shared lessons or media objects, must be kept as long as necessary after creations, a repository of learning objects is then required not only for making them available for search and reuse, but also enabling a means for an authoring application to publish its resources. In this regards, Digital Content Repository (DCR) that works on generic metadata specifications (e.g. Dublin Core or MARC) for documenting general contents is more suitable than the counterpart of Learning Object Repository (LOR) [8] that is rather restricted to specific metadata standards (e.g. IEEE LOM) for describing learning contents. Therefore, the DCR will be selected in our proposed architecture so that a wider range of content types (e.g. photograph and AV files) can be accommodated.

\section{Linked Data Service}

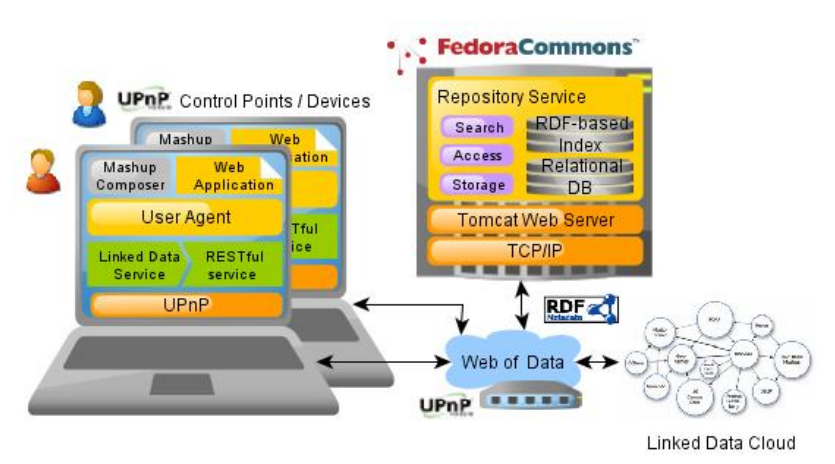

Fig. 3. Demonstration setup.

In many cases, learning resources may be instantly created on-demand, such as snapshots with some contextual contents (e.g. the current geo-location) or sensory data in a timely fashion. It is certain that storing these contents is not so useful at the central repository, but would rather be better at the local for immediate response, e.g. during the collaborative activity. In this regards, linked data service will 
be required for exposing them in a Linked Data-compliant way. By means of RDF URIs, linked data resources from different sources can be discovered, shared and consumed by the other peer devices. This will be convenient if an authoring application can be involved for enabling semi-automatic composition and automatic generation of Web Mashups [9], which is quite popular for modern Web 2.0 applications.

\section{PRototype AND A Simple CASE}

This section introduces the general architecture of our prototype and describes a simple case of Mashup application, in order to demonstrate the linked-data usage and integration of learning data from different sources.

\section{A. Prototype Architecture}

A simple implementation for realizing ubiquitous learning services in a P2P manner can be illustrated in Fig. 3. The ad-hoc network is supported over the Wi-Fi network infrastructure. All computers are turned into UPnP-capable devices by enabling the UPnP support in Windows Platform. It is noticed that the semantic-based repository is not UPnP-compliance, but works in compatibility with UPnP devices via standard TCP/IP protocol. Indeed, this repository is a computer running Apache Tomcat Web server and Fedora Commons [10] open source software (which will be called Fedora hereafter) for providing RDF-enabled repository in our mobile learning environment.

Due to many distinct features, Fedora server allows users of UPnP devices to access the semantic repository for searching, adding, modifying and retrieving digital objects together with their metadata, in many different ways. For instance, by means of SOAP-based or RESTful Web services, developers of Web applications can have automatic association with Fedora in several programming languages. Alternatively, by using Web browser, users can interface manually to administrative module of Fedora, or issue query statements written in SPARQL language and view the results in response accordingly.

\section{B. Mashup Application}

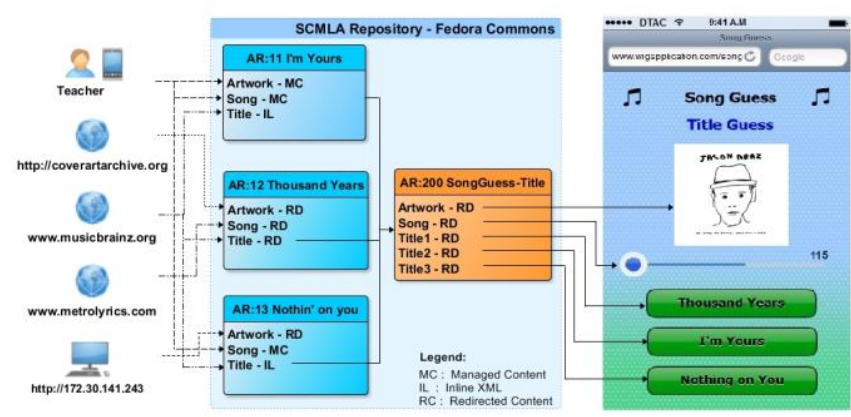

Fig. 4. Example quiz for guessing the song title.

To make the proposed system more comprehensible, we conducted a simple example that illustrates how learning resources (such as assessment quizzes) can be generated by a combined set of RDF data collecting from different Linked Data sources. A simple example as shown in Fig. 4 has been implemented, which is slightly more complex than the work in [11]. It is a "Song Guess" Application that displays an artwork of song that is currently playing and the user are about to pick up the correct song title from the displayed list. According to our design, each element is generated from a digital object that aggregates a number of contents (known as datastreams in the Fedora context) from multiple sources locating either in the Fedora repository or from the external data sources referenced by URLs as they can be noticed in the left part of Fig. 4.

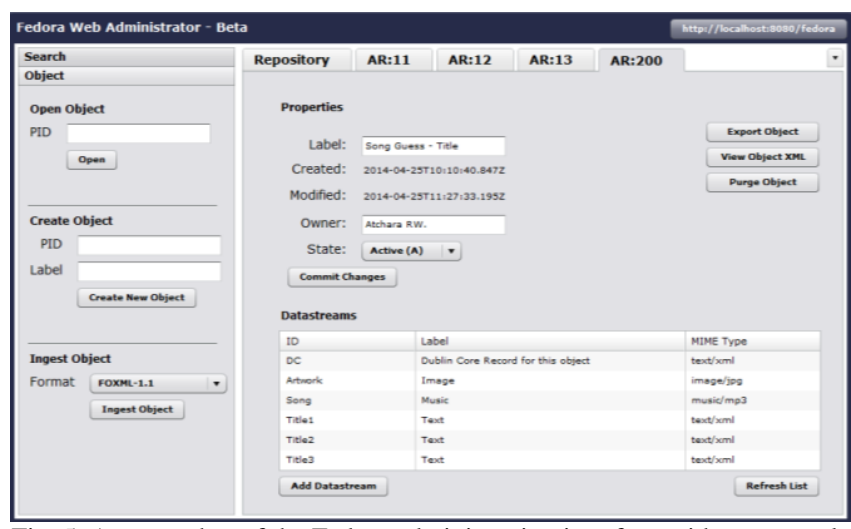

Fig. 5. A screenshot of the Fedora administration interface with an example of digital object.

In our design, the primary data objects are AR:11, AR:12, and AR:13 and are specified the handle or linkage to actual resources in different manners. For example, the datastream of AR:11 that has the type of "Managed Content (MC)" will store the content (e.g., jpg file of Artwork) in the Fedora repository, but the type of "Inline XML (IL)" will be stored the element of data source as part of XML structure. In contrast, the datastream of AR: 12 that have the type of "Redirected Content (RC)" will only deliver the content to the client without any mediation by the Fedora. It should be noticed that the secondary data object (AR: 200) is designed particularly to provide the linkages to those primary ones for constituting the structure of RDF data that will be promptly used in the quiz application. In Fig. 5, these linkages can be easily noticed in the bottom part of the Fedora administration interface.

Hence, by means of different types of datastreams, the Fedora repository can be mandated to support different ways of manipulation and relationship establishment among content objects to suit our needs. In this regard, it will become easy and flexible for lesson developers to rely on the Fedora repository for storing resulted linked data of Mashup composer or some other applications.

\section{Miscellaneous}

To summarize, the prototype implementation described in the previous sub-section is a preliminary evaluation for our pilot application, which takes Semantic Web and Linked Data to facilitate for quiz-like question development by taking Linked Data cloud as a source of knowledge. In general, this development process may become a tedious work, since it requires the SPARQL Query Language to access data from several Linked Data resources of interest. However, in our system, this is much easier through the use of administrative Web Interface (e.g. as examples shown in Fig. 5 to Fig. 7), which obviously does not need any programming skill for creating, viewing, or managing digital 
learning objects at all. In addition, by deploying Fedora at the backend of digital repository, user application can be accessed to powerful functionalities either via SOAP Service or REST API using programming language of choice. This is far more complex in traditional system, where SPARQL is needed, and the other software for serving RDF data over HTTP (e.g. Apache's Jena Fuseki) is inevitable.

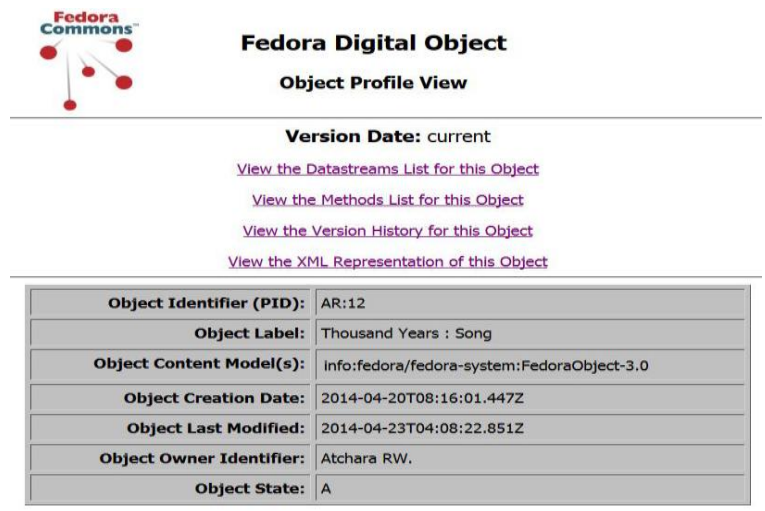

Fig. 6. A screenshot of the Fedora administration interface for viewing a digital object profile.

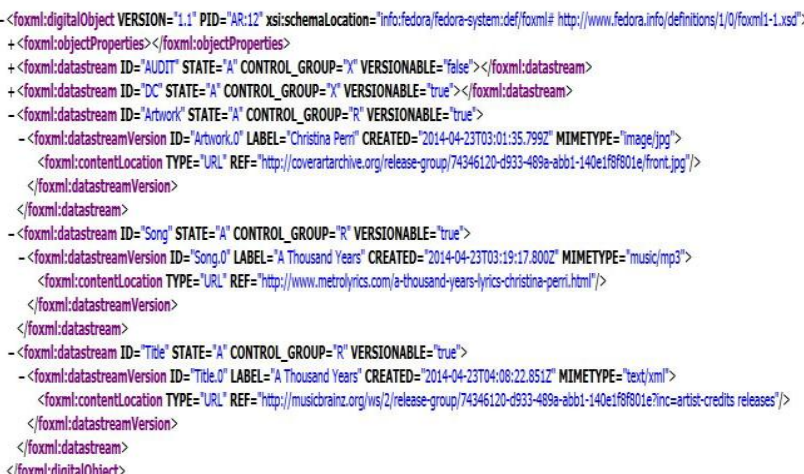

Fig. 7. A screenshot of the Fedora administration interfacewith a XML list of digital object.

\section{RELATED WORKS}

There exist some similar efforts within the research field of our research. For example, Pathmeswaran and Ahmed [12] developed a prototype of $S W m L O R$ system, which attempted to provide a better facilitation of mobile learning resources by accompanying standard learning objects with ontology-based semantic contents. Although their architectural framework provides the same functionalities as that of our works, it contains three major drawbacks; a) facilitating the poor support for collaborative learning community between mobile devices in the local network, due to the intrinsic limitation of client-server based learning environment, b) overlooking the use of Linked Data approach for exposing learning resources and allowing them to be reused or shared in the simplified manner at mobile devices, and c) ignoring the exploitation of open source software for digital repository tool (i.e. Fedora in our case), which can immensely simplified the programming tasks. Li, Chen and Huang [13] takes the advantages of Fedora open source software as well for building a prototype of learning resource management and sharing system (called SULOMS) with adaptive capabilities, which is claimed to be suitable for mobile learning. Our work takes a broader view of sharing semantic learning objects via the Web of Linked Data.

To provide a better support for collaborative learning environment, several works attempts to bring P2P technology into consideration. For example, Jin et al. [14] described a peer-to-peer based learning environment over the Internet, named APPLE, which has a combination of $\mathrm{P} 2 \mathrm{P}$ overlay network, Internet broadcast system, and grid technology, and reported satisfactory results in educational experiments with general computers. Compared to this research work, our system is much simpler and does not require any burden of $\mathrm{P} 2 \mathrm{P}$ routing algorithms, due to the dominant feature of UPnP protocol stack and service-oriented architecture involved. Yen et al. [15] described their developed system called iServe that is Linked Data-driven and works on service-oriented architecture and claimed its efficiency in resolving the problem of interoperability issues due to various metadata standards of learning objects in a distributed and heterogeneous environment. Unlike our system, the iServe system lacks the support for P2P operations in the scope of this work. The summarized view of the aforementioned comparison of related systems can be seen in Table I.

TABLE I: SUMMARIZED COMPARISON OF RELATED WORKS

\begin{tabular}{lcccc}
\hline \hline System & $\begin{array}{c}\text { P2P } \\
\text { Support }\end{array}$ & $\begin{array}{c}\text { Linked Data } \\
\text { Approach }\end{array}$ & $\begin{array}{c}\text { SOA } \\
\text { Principle }\end{array}$ & $\begin{array}{c}\text { Fedora } \\
\text { Engine }\end{array}$ \\
\hline SWmLOR [12] & No & No & No & No \\
\hline SULOMS [13] & No & Yes & No & Yes \\
\hline APPLE [14] & Yes & No & No & No \\
\hline iServe [15] & No & Yes & Yes & No \\
\hline Our work & Yes & Yes & Yes & Yes \\
\hline \hline
\end{tabular}

\section{CONCLUSION}

This paper introduced UPnP technology in the ubiquitous learning area and suggested the lightweight semantic web of Linked Data approach to publish learning data on the Web so that other peer devices can access and interpret the data using merely standard Web technologies. Comparing with traditional mobile and ubiquitous learning, our system has advantages as follows: 1) It can discover any networked UPnP devices automatically and provides the instant support of peer-to-peer networking; 2) It enables the web-driven technology and thus allows for the Linked Data to be utilized across the network domain via the Internet if the security policy can be met; 3 ) It does not need any tedious operations for accessing the elements of shared data, which is inevitable in many cases of metadata standards for learning object interoperability. In addition, we show the linked-data usage and integration of learning data from different sources through a simple quiz example, which can be potentially led towards more realistic or advanced explorations, which is currently an ongoing work.

\section{REFERENCES}

[1] S. Martin, G. Diaz, I. Plaza, E. Ruiz, M. Castro, and J. Peire, "State of the art of frameworks and middleware for facilitating mobile and ubiquitous learning development," J. of Systems and Software, vol. 84, no. 11, pp. 1883-1891, November 2011.

[2] UPnP Device Architecture version 1.1, UPnP Forum, Oct 2008.

[3] C. Bizer, T. Heath, and T. Berners-Lee, "Linked data - The story so far," Int. J. on Semantic Web and Information Systems, vol. 5, no. 3, pp. 1-22, 2009. 
[4] H. Shin, "Challenges and the solutions for multimedia metadata sharing in networks," in Multimedia - A Multidisciplinary Approach to Complex Issues, I. Karydis, Ed., 2012, pp. 263-276.

[5] M. Svensson, A. Kurti, and M. Milrad, "A linked data approach for enriching emerging learning objects with contextual metadata," presented at Fourth European Conference on Technology Enhanced Learning, Nice, France, September 29-October 2, 2009.

[6] B. Lund. (November 2011). Integration of UPnP with HTML5. [Online]. Available: http://html5.cablelabs.com/upnp/ html5-upnp-integration.html.

[7] M. Birbeck. (June 2009). Introduction to RDFa. [Online]. Available: http://alistapart.com/article/introduction-to-rdfa.

[8] R. Klemke, S. Ternier, M. Kalz, and M. Specht, "Implementing infrastructures for managing learning objects," British Journal of Educational Technology, vol. 41, no. 6, pp. 873-882, 2010.

[9] H. H. Hoang, T. N.-P. Cung, D. K. Truong, D. Hwang, and J. J. Jung, "Semantic Information Integration with Linked Data Mashups Approaches," Int. J. of Distributed Sensor Networks, vol. 2014, p. e813875, April 2014.

[10] C. Lagoze, S. Payette, E. Shin, and C. Wilper, "Fedora: an architecture for complex objects and their relationships," Int. J. on Digital Libraries, vol. 6, no. 2, pp. 124-138, April 2006.

[11] G. Á. Rey, I. Celino, P. Alexopoulos, D. Damljanovic, M. Damova, N. $\mathrm{Li}$, and V. Devedzic, "Semi-automatic generation of quizzes and learning artifacts from Linked Data," presented at the Linked Learning 2012: 2nd Int. Workshop on Learning and Education with the Web of Data, Lyon, France, April 17, 2012.

[12] R. Pathmeswaran and V. Ahmed, "SWmLOR: Technologies for developing semantic web based mobile learning object repository," The Built \& Human Environment Review, vol. 2, no. 1, Jul. 2011.

[13] Y. Li, Z. Chen, R. Huang, and M. X. Cheng, "An Integrated Learning Resource Management System with Web Services," in Proc. Int. Conf. on New Trends in Information and Service Science, 2009, pp. 863-868.

[14] H. Jin, Z. Yin, X. Yang, F. Wang, J. Ma, H. Wang, and J. Yin, "Apple: A novel P2P based e-learning environment," in Distributed Computing - IWDC 2004, A. Sen, N. Das, S. K. Das, and B. P. Sinha, Eds., Springer Berlin Heidelberg, 2005, pp. 52-62.

[15] H. Q. Yu, S. Dietze, N. Li, C. Pedrinaci, D. Taibi, N. Dovrolls, T. Stefanut, E. Kaldoudi, and J. Domingue, "A linked data-driven \& service-oriented architecture for sharing educational resources," presented at the 1st Int. Workshop on eLearning Approaches for Linked Data Age (Linked Learning 2011), 8th Extended Semantic Web Conference (ESWC2011), Heraklion, Greece, 2011.

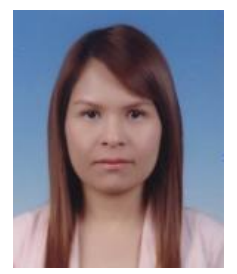

Atchara Rueangprathum was born in Songkhla province, Thailand on September 8, 1977. She completed a bachelor degree in business administration (information systems) from Rajamangala Institute of Technology, Thailand in 2000 and a master of science degree in management of information technology from the Prince of Songkla University in 2005. Currently, she is a Ph.D student of the Department of Computer
Engineering, Faculty of Engineering, Prince of Songkla University, Thailand.

She was a lecturer in the Department of Computer Science, Faculty of Science and Information Technology, Suratthani Rajabhat Universtiy, Suratthani. Some of her publications such as: e-learning in Thailand - a survey of current situation and trend. International Conference on Teacher Professional Development Searching for New paradigms, Agendas and Network (ICONTPD2009). Her current research interests include mobile learning and semantic web technology.

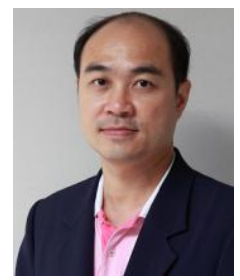

Somchai Limsiroratana was born in the southern of Thailand on December 20, 1969. He holds a bachelor's degree in electrical engineering from the Faculty of Engineering, Prince of Songkla University, Thailand, since 1991. master and doctor degrees in agricultural science from the Division of Environmental Science and Technology, Kyoto University, Japan, since 2000 and 2005 , respectively, with the thesis topics related to detect the positions of papaya fruits in natural conditions.

He has been working as a senior lecturer of the Department of Computer Engineering, Faculty of Engineering, Prince of Songkla University (Hat Yai campus) since 1991. He taught/teaches subjects such as the Introduction to Computer Programming, Computer Architecture, Object-Oriented Analysis and Design, Software Engineering, Image Processing, Artificial Intelligent (AI), etc. His research interests are agricultural image processing, medical image processing, digital watermarking and AI.

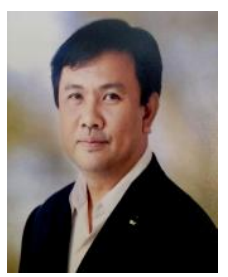

Suntorn Witosurapot is an assistant professor in Department of Computer Engineering, Faculty of Engineering, in Prince of Songkla University (PSU), HatYai, Thailand. He received the bachelor and Master degrees in electrical engineering from PSU, Thailand and Ph.D. degree from Swinburne University of Technology, Melbourne, Victoria, Australia, with the thesis topics related to resolving network resource competition in the Internet.

His research interests include web engineering and applications, model-based software engineering, and management of information technology. Currently, most of his research work revolves around information engineering in smart home network environment and smart grid infrastructure 